\title{
Preliminary Report on Infrared Radiometric Measurements from the Mariner 9 Spacecraft
}

\author{
H. H. KIEFFER \\ Department of Planetary and Space Science, University of California \\ Los Angeles, California 90024 \\ S. C. Chase, JR. \\ Santa Barbara Research Center, Goleta, California 99017 \\ E. Miner \\ Jet Propulsion Laboratory, California Institute of Technology \\ Pasadena, California 91103 \\ G. MÜNCh AND G. Neugebauer \\ Division of Physics, Math, and Astronomy, California Institute of Technology \\ Pasadena, California 91109
}

\begin{abstract}
Preliminary 10- and 20- $\mu \mathrm{m}$ brightness temperatures of Mars are presented. More than $35 \%$ of the Martian surface was observed with a resolution better than $100 \mathrm{~km}$. On the whole, the results confirm the thermal properties derived from the Mariner 6 and 7 radiometers, although the temperatures, on the average, were cooler and shifted with respect to the Martian day during the dust storm. Thermal inertias and radiometric albedos were derived for many areas; no clear correlation exists between these properties. Thermal structure again was found at the spatial limit of the radiometer; no cases were found in which it was necessary to invoke internal heat sources.
\end{abstract}

The infrared radiometer experiment carried on Mariner 9 was intended to thermally map the surface of Mars at 10 and $20 \mu \mathrm{m}$ with a spatial resolution unobtainable from the earth and to extend the coverage achieved by the radiometers aboard Mariner 6 and 7. In 1969 approximately $2 \%$ of the Martian surface was observed by the radiometers with a linear resolution of, at best, $50 \mathrm{~km}$ [Neugebauer et al., 1971]. On the Mariner 9 mission, more than $35 \%$ of the surface was observed with a spatial resolution of $100 \mathrm{~km}$ or less; many areas were observed with a resolution as good as $15 \mathrm{~km}$.

From the Mariner 6 and 7 observations, which covered a limited range of latitudes and local times, average thermal properties of the surface were deduced; a best fit to the bulk of the data was achieved with a bolometric albedo $A$ around 0.3 and a thermal inertia $I=(k \rho c)^{1 / 2}$ of 0.006. Following Neugebauer et al. [1971],

Copyright (C) 1973 by the American Geophysical Union. the following units will be used throughout this work and usually omitted from the text: thermal conductivity $k$, cal $\mathrm{cm}^{-1} \mathrm{sec}^{-1}{ }^{\circ} \mathrm{K}^{-1}$; specific heat $c$, cal $\mathrm{gm}^{-1}{ }^{\circ} \mathrm{K}^{-1}$; density $\rho, \mathrm{gm} \mathrm{cm}^{-3}$; thermal inertia $I=(k \rho c)^{1 / 2}$, cal $\mathrm{cm}^{-2} \mathrm{sec}^{-1 / 2}$ ${ }^{\circ} \mathrm{K}^{-1}$. The thermal model using these values has been used as the basis for a preliminary comparison with the 1971 data; the model will be referred to as the Mariner 69 thermal model.

Although the average thermal properties deduced in 1969 adequately represented most of the observed Martian thermal structure, some large areas, especially Hellas, were systematically different and required inertias as high as $\mathbf{0 . 0 1 0}$. There was a clear correlation of temperatures with the classical features on Mars. In addition, a few local thermal features appeared at the limit of the spatial resolution of the radiometer. In particular, one such feature was tentatively identified with a crater in Deucalionis Regio.

Earth-based infrared observations of Mars 
before the Mariner 6 and 7 missions were reviewed by Neugebauer et al. [1971]. Although several observational earth-based programs have been carried out since that time relating to specific Martian problems, no global study of the thermal properties has been published.

A single-channel radiometer covering the wavelength range $8-40 \mu \mathrm{m}$ was incorporated in the Soviet spacecraft Mars 1 and 2. Preliminary reports show apparent agreement between the Russian and Mariner 9 data [Chase et al., 1972; Pravda, 1972].

In this paper a preliminary summary of some aspects of the radiometer measurements will be presented. About $1.5 \times 10^{3}$ individual measurements of the surface temperature of Mars were obtained in each of the two radiometric channels. The data, as received, contain transmission bit errors that must be eliminated for computer processing. Furthermore, detailed analyses of instrumental properties necessary to delineate effects of the off-axis response of the radiometer have not been completed. As a result, the data treated in this paper are limited to those taken far enough from the Martian limb for any corrections for the extended field response to be less than $10 \%$ in flux. In effect, this excludes observations with emission angles greater than $60^{\circ}$. Primarily, this limitation postpones detailed evaluation of the absolute value and phase dependence of emissivity; the north and south polar caps will also be reserved for future discussion. All temperatures presented will necessarily be brightness temperatures $T_{10}$ and $T_{20}$ at either 10 or $20 \mu \mathrm{m}$.

The discussion of the data will be divided into three sections, each concerned with thermal features with different linear scales. The planet as a whole defines a natural upper scale. The classical features seen on the planetary surface define the large-scale structure. Finally, the instrumental resolution of $15-100 \mathrm{~km}$ defines the smallest meaningful scale. The division is clearly arbitrary, and only a minimal attempt at a rigorous consistency in following it has been made.

\section{INSTRUMENTATION}

The infrared radiometer flown on Mariner 9 has been described by Chase [1969] and Chase et al. [1970] and reviewed by Neugebauer et al. [1971]. The only essential modification from the instrument of the 1969 mission was the reduction from $0.7^{\circ}$ to $0.5^{\circ}$ of the field of view of the $10-\mu \mathrm{m}$ channel.

During the 1971 mission, data were taken every $1.2 \mathrm{sec}$ when the instrument platform was nominally pointed at Mars; as observations ranged from periapsis to apoapsis, the linear scale of the area observed ranged from about 15 to about $1000 \mathrm{~km}$. The radiometer was rigidly boresighted with the television cameras, and initial orbital elements were selected to maximize television contrast. As a result, the coverage on the planet was nonuniform with respect to local times. For example, near the end of the mission the data obtained from latitudes of $-30^{\circ}$ to $+40^{\circ}$ were concentrated in a band of local times (with 0000 at midnight) between 1300 and 1700 ; in contrast, equatorial data in the early parts of the mission were concentrated in the two bands, 0500-0900 and 1500-1800.

Rapid scans onto the bright limb of Mars show the existence of a significant extended field response of the radiometer. The instrument sensitivity falls off rapidly at the edges of the nominal fields of view to less than $10 \%$ at $0.5^{\circ}$ and $0.6^{\circ}$ from their center for the 10- and $20-\mu \mathrm{m}$ channels, respectively. However, the sensitivity decreases slowly and is less well known at larger angles. Approximately 10 and $40 \%$ of the total response come from the area beyond $1.4^{\circ}$ from the center for the 10 - and $20-\mu \mathrm{m}$ channels, respectively. The data discussed in this paper have not been corrected for this effect. As stated, however, a first-order analysis has been used to exclude the small fraction of the data where the correction is estimated to be larger than $10 \%$ in flux. As the central field of view is well defined, the spatial resolution used in analysis of local features is little affected by the extended response. On the basis of prelaunch calibrations and comparisons with the internal reference calibrator, the brightness temperatures presented have an absolute accuracy of better than $5^{\circ} \mathrm{K}$ near $250^{\circ} \mathrm{K}$; the resolution of both the 10 - and $20-\mu \mathrm{m}$ temperatures is better than $1^{\circ} \mathrm{K}$. The internal calibration was very stable throughout the mission.

\section{Planetwide Properties}

Observations. The smoothed $10-\mu \mathrm{m}$ brightness temperatures observed at three epochs in 


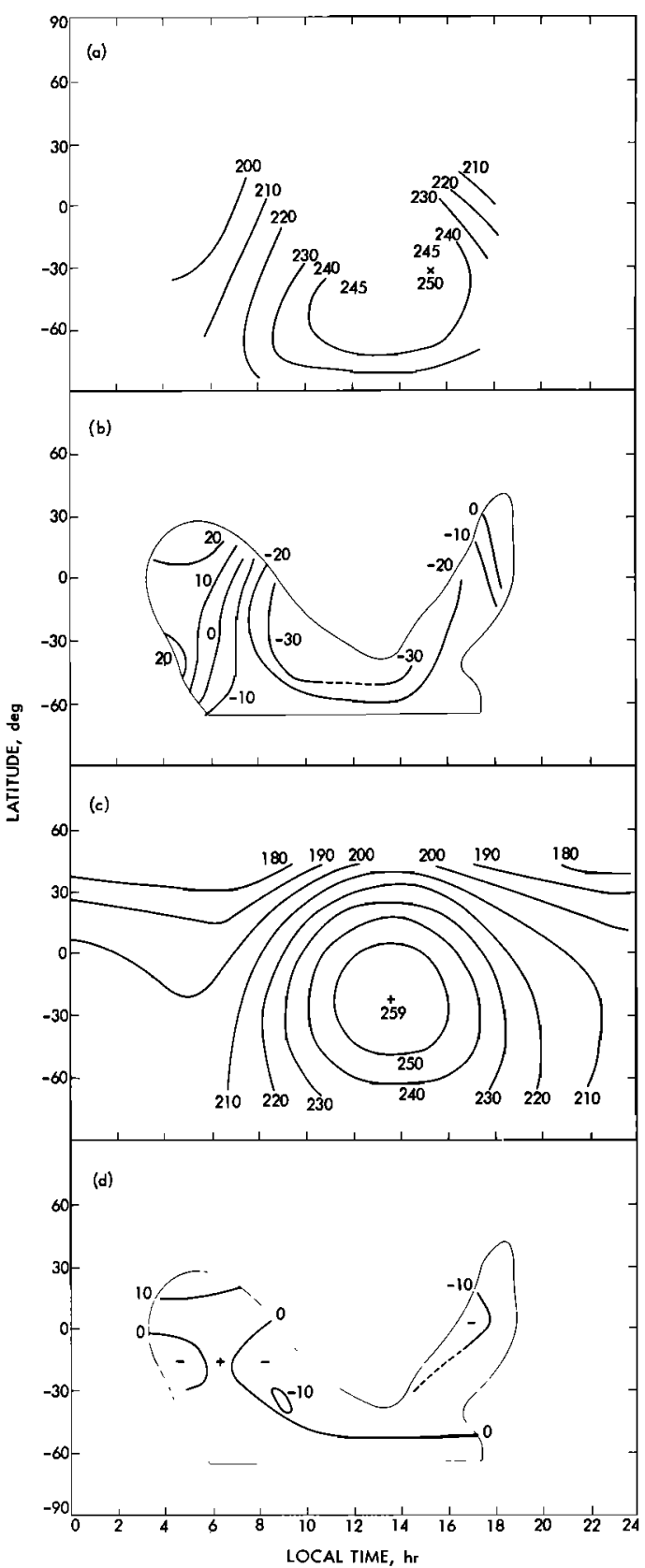

Fig. 1. Observed and model temperatures. The following data are shown, averaged over bins of $5^{\circ}$ latitude by half-hour local time. (a) Observed $10-\mu \mathrm{m}$ brightness temperatures, averaged over 60 revolutions centered around December 9, 1971. The subsolar latitude is $-19^{\circ}$. (b) Observed 10- $\mu \mathrm{m}$ temperatures minus those computed by using the Mariner 69 thermal model. (c) Temperatures for the model using parameters that best-fit the observed data from latitude $-70^{\circ}$ to $+40^{\circ}$. (d) Observed $10-\mu \mathrm{m}$ temperatures minus those obtained from the best-fit model. the mission are shown in Figures 1-3. In presenting the general picture of the planetary temperatures, the data are considered as a function of latitude and local time only, and variations with longitude have been ignored.

At the beginning of the mission, temperature variations across the planet were small, and the

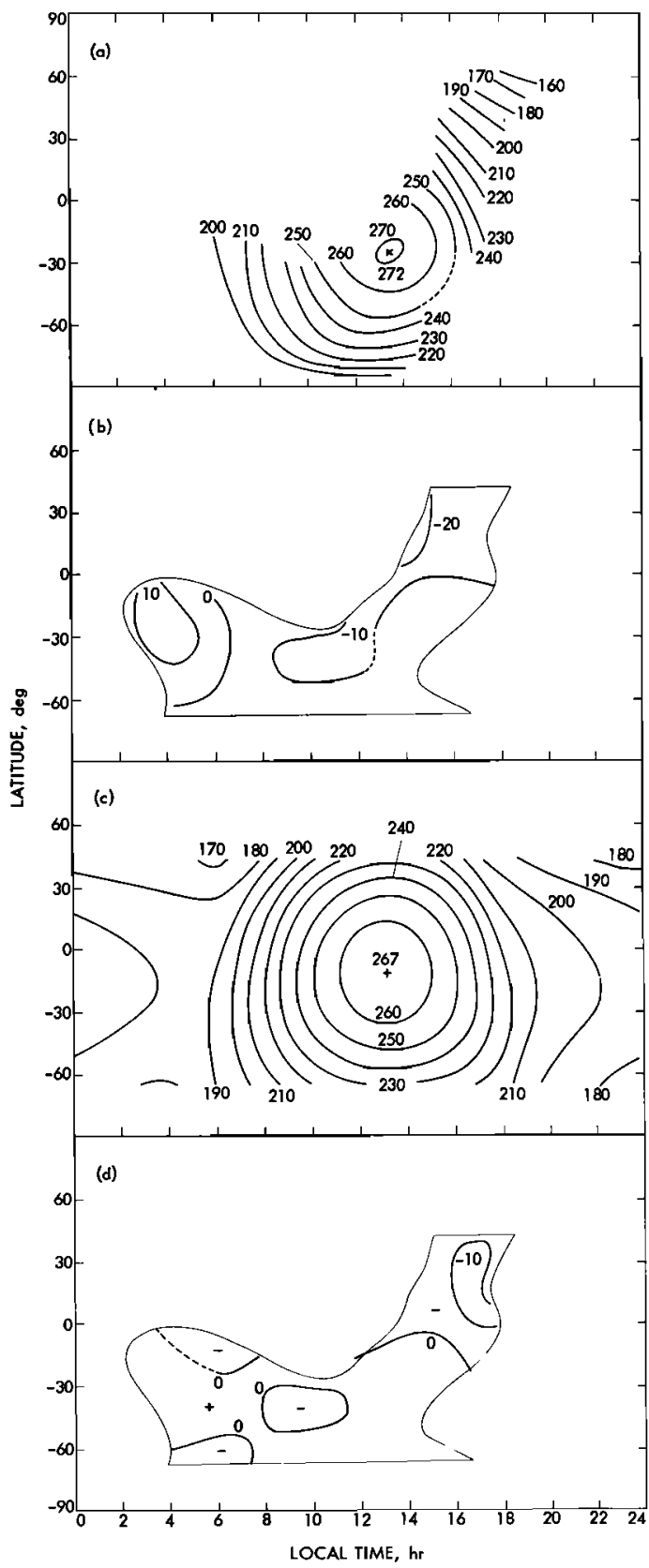

Fig. 2. Same as Figure 1 for data centered on January 18,1972 , with subsolar latitude of $-13^{\circ}$. 


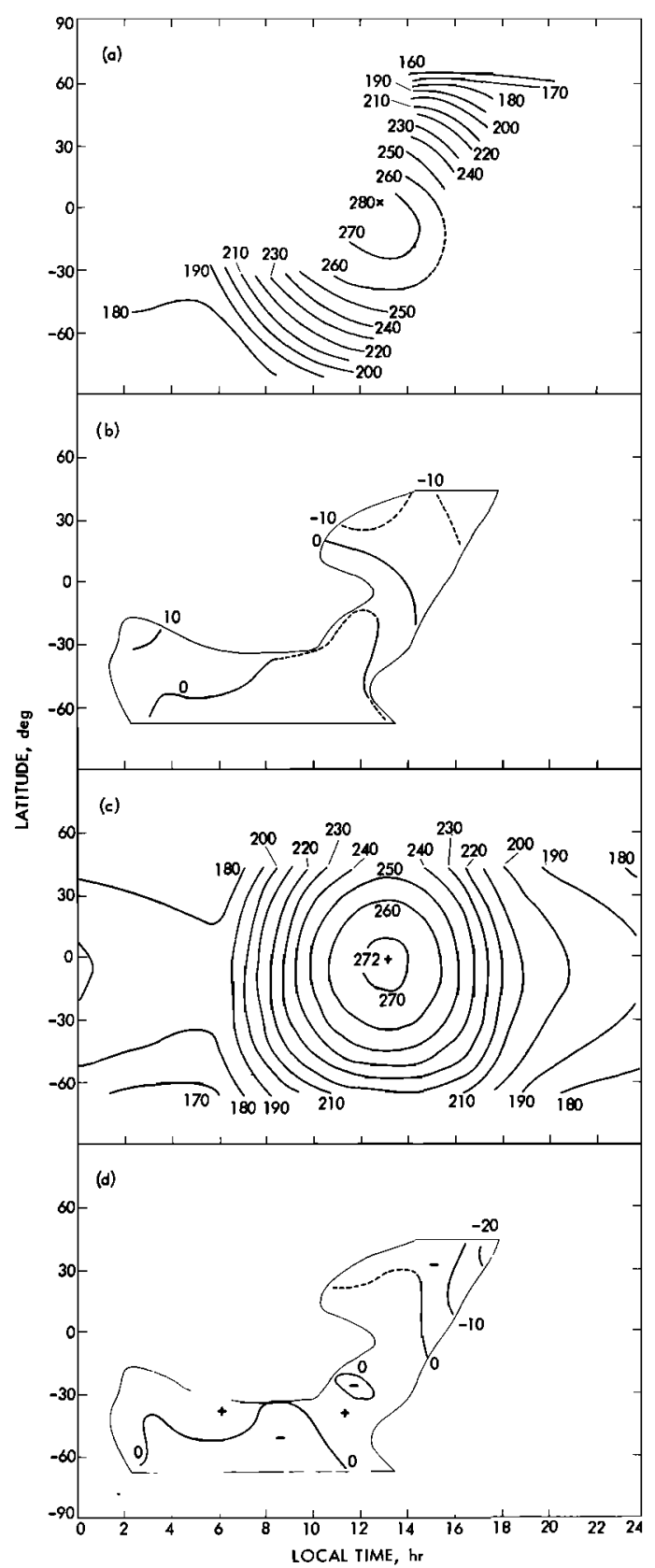

Fig. 3. Same as Figure 1 for data centered on February 17, 1972, with subsolar latitude of $-6^{\circ}$.

maximum temperature occurred at latitudes well south of the subsolar latitude. Near latitude $-40^{\circ}$, the peak temperatures occurred almost 3-4 hours after noon. The temperature pattern differed radically from that predicted by calcu- lations using the physical parameters derived from the Mariner 69 data. The planet looked smooth thermally, and it was evident that the temperature changes expected from the surface albedo variations were absent. The one exception, other than the southern polar cap, was the set of dark areas observed in the early television pictures and later identified as the volcanoes along the Tharsis ridge. These dark areas had temperatures clearly different from those of the surrounding areas and approaching those predicted by the Mariner 69 thermal model.

As the mission progressed, the temperature contrast across the planet increased, and the position of maximum temperature shifted toward the subsolar latitudes and to a time one hour after noon. The maximum temperature increased through the mission, although Mars moved farther from the sun in its eccentric orbit. The highest observed temperature was $285^{\circ} \mathrm{K}$.

Thermal models. Analysis of the radiometer data, whether on the global or localized scale, has always been made with respect to a basic thermal model described by Neugebauer et al. [1971]. Fundamentally, the model consists of a spherical, homogeneous, conductive solid, heated by insulation and radiating to space. The free parameters defining the model are the bolometric albedo $A$ and the thermal inertia $I$, hereafter frequently referred to as simply albedo and inertia. The effect of atmospheric radiation is included explicitly only by incorporating a constant atmospheric back radiation equal to 0.02 of the noon solar flux at that latitude.

The effects on the model surface temperatures of varying the albedo and inertia are shown in Figure 4. Increasing albedo decreases the temperatures at all times, the effect being largest near midday and diminishing steadily until dawn. Increasing inertia decreases the amplitude of the diurnal variation and increases the delay of peak temperatures after noon; it also increases the average temperature slightly. Most significantly, for representative ranges of albedo and inertia the derivatives of Figure 4 show that data obtained around local noon depend strongly on the bolometric albedo. In contrast, the properties of the data taken just before sunrise, the so-called predawn data, depend almost exclusively on the assumed thermal inertia. The model diurnal variation for Martian 


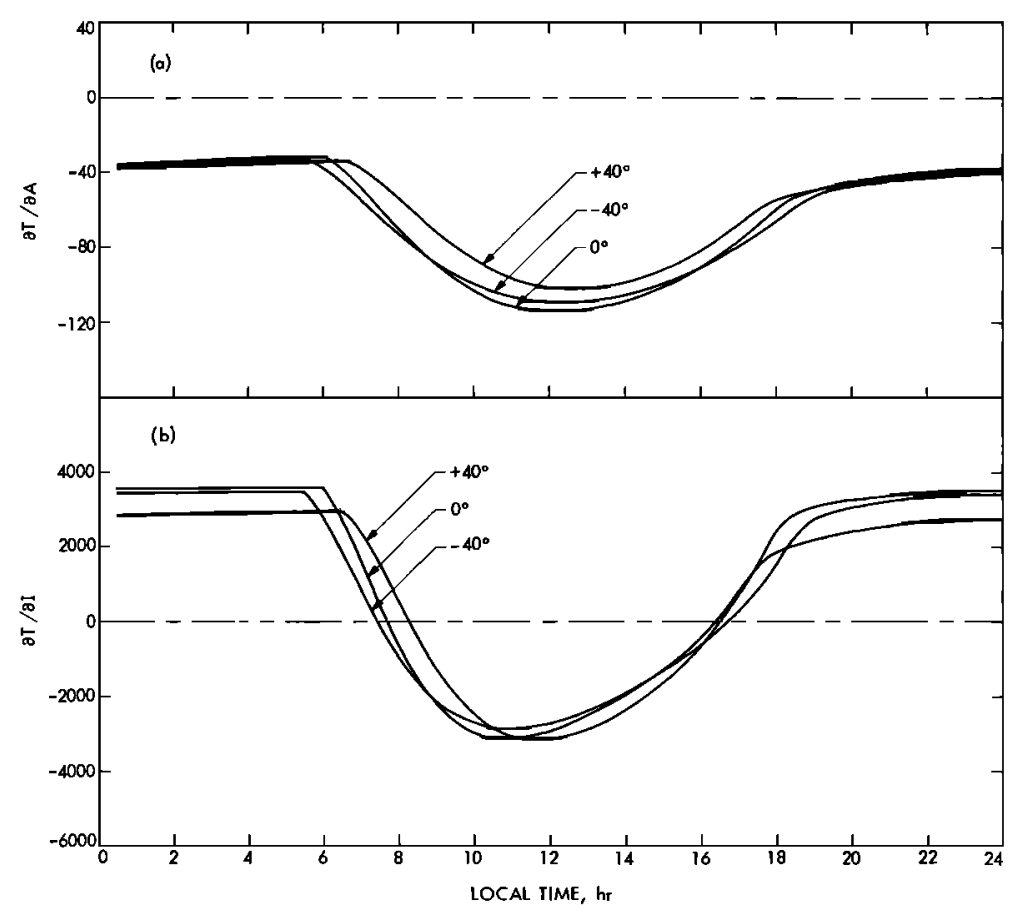

Fig. 4. Dependence of the model temperatures on ( $a$ ) albedo and $(b)$ inertia. Curves are shown for three latıtudes on January 28, 1972. Notice that $\partial T / \partial A$ is always negative, the maximum absolute value near noon diminishing rapidly until sunset and then steadily at a lesser rate until dawn. In contrast, $\partial T / \partial I$ has a small positive average over the day, with large negative values near midday and nearly constant positive values at night. The magnitude of $\partial T / \partial I$ decreases as the lengths of day and night become unequal.

albedos and inertas is primarily a function of the assumed thermal inertia.

Within the ranges $0.2 \leq A \leq 0.4$ and 0.005 $\leq I \leq 0.010$, temperatures have been calculated by using a linear expansion and the derivatives in Figure 4. This procedure has been verified to be accurate to within $2^{\circ} \mathrm{K}$ by comparison with a limited set of direct integrations.

A free parameter of the thermal model is the emissivity in the 10 - to $20-\mu \mathrm{m}$ region, which is incorporated as a boundary condition. In the model calculations made to analyze the Marmer 9 data, an emissivity $\epsilon$ of 1.0 wiss assumed, thus facilitating direct comparison with the observed brightness temperatures. In the 1969 model an emissivity of 0.9 was assumed, but the observed Mariner 9 brightness temperatures have nevertheless been compared directly with the model surface kinetic temperatures. Characteristically, the Mariner 69 model temperatures are too warm by $(1-\epsilon)^{1 / 4} T \approx$ $0.02 T^{\circ}$; this small discrepancy does not signifi- cantly affect comparison between observations at different times or places.

Finally, the regular radiometer observations lasted from mid-November 1971 through midMarch 1972. During that time, the Mars solar distance increased from 1.41 to $1.52 \mathrm{AU}$, and the subsolar latitude changed from $-23^{\circ}$ to $0^{\circ}$. The peak temperature predicted by the thermal model with the Mariner 69 set of thermal parameters correspondingly decreased from $292^{\circ}$ to $274^{\circ} \mathrm{K}$.

Discussion. The observed $10-\mu \mathrm{m}$ brightness temperatures $T_{1 \mathrm{n}}$ for the latitude range $-70^{\circ}$ to $+40^{\circ}$ have been fit, in a least squares sense, to the thermal model calculated for the appropriate season. All data obtained in a 42 -sec period were averaged; then the averaged local time and latitude were used to sort $T_{30}$ into bins of $5^{\circ}$ latitude and half-hour local time. Each bin was weighted by the square root of the number of observations at that location. By using a starting model temperature $T_{m}$ and 
$\partial T_{m} / \partial A$ and $\partial T_{m} / \partial I$ as shown in Figure 4 , a least squares fit model temperature $T_{L}$ was found. Because possible large data errors caused by bit errors in transmission could affect this fit, any points with $\left|T_{10}-T_{L}\right|>15^{\circ} \mathrm{K}$ were deleted in this preliminary analysis, and a second fit made. A program is in progress to identify and correct or eliminate all data affected by significant bit errors.

The results of the fit are included for three epochs of the mission in Figures 1-3. It is obvious that, even late in the mission when effects of the dust storm had diminished, consistent departures from the best-fit models exist. The observed temperatures are generally low with respect to the best-fit models at latitudes from $-70^{\circ}$ to $-40^{\circ}$ and near $1600 \mathrm{LT}$ for all latitudes observed. This was particularly evident on revolutions 120-130 in January 1972, when a set of special observations was incorporated, during a gap in the television mapping sequence, to cover all the accessible range of local times at latitudes from $+10^{\circ}$ to $+40^{\circ}$. This rapid drop in temperature is not reproducible with the thermal model and may indicate an increased thermal coupling between the surface and the atmosphere in the late afternoon.

The time variations of albedo and inertia derived for the best planetwide fit to the thermal model are shown in Figure 5. The derived parameters early in the mission are not meant to be taken as physically meaningful but rather as indications of the effect of the dust storm and its smooth progression with time. Also, the results for the early orbits are far from the region for which the model derivatives were evaluated and hence are not accurate. The oscillations in inertia observed during the earlier part of the mission apparently are due to statistically significant variations in the predawn temperatures. The inertia oscillations have a period of 40 revolutions, which corresponds to the beat period of the spacecraft periapsis point and the Martian longitude. The times of minimum inertia were obtained when many of the predawn observations were at longitudes near $110^{\circ}$, a region significantly colder than other longitudes before sunrise.

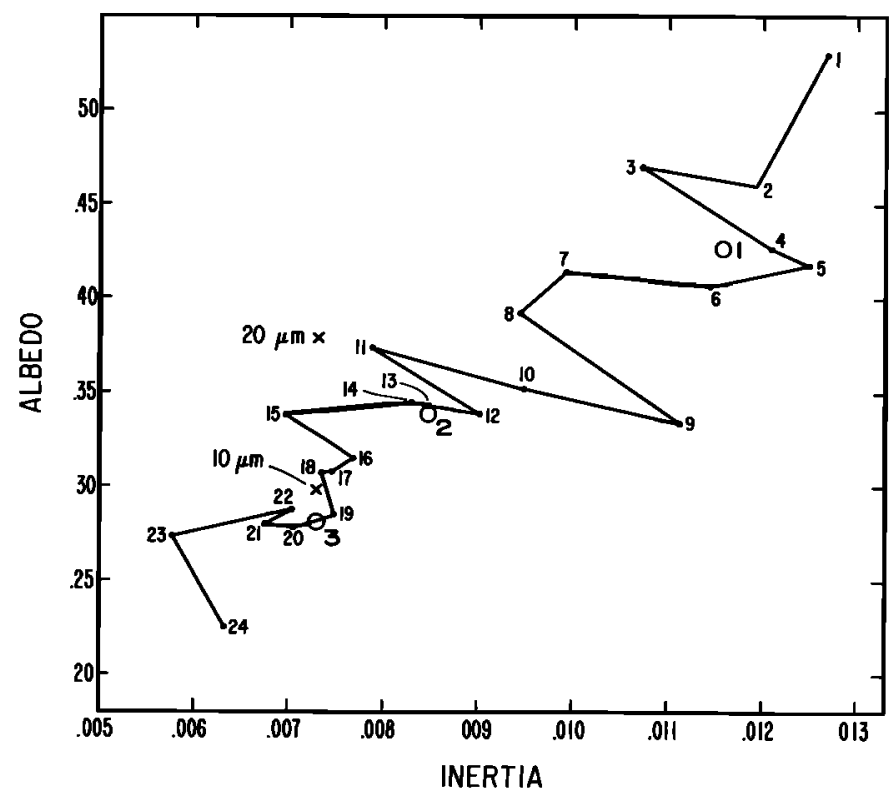

Fig. 5. The variation through the mission of apparent albedo and inertia as derived from the best-fit models over groups of 10 revolutions. The large oscillations have a period of about 40 rcvolutions, corresponding to the time requircd for the radiometer swath to cover the planet once. The last, two points are different from the converging trend of previous orbits and may be due to errors in the spacecraft-planet geometry. The numbers by the solid dots indicate sequential groups of 10 orbits. The three numbers in the open circles show the values of albedo and inertia derived from the data of Figures 1, 2, and 3 . The crosses indicate the values arrived at by using the Mariner 6 and 7 data. 
Kieffer et AL.: MARINer 9 Mission

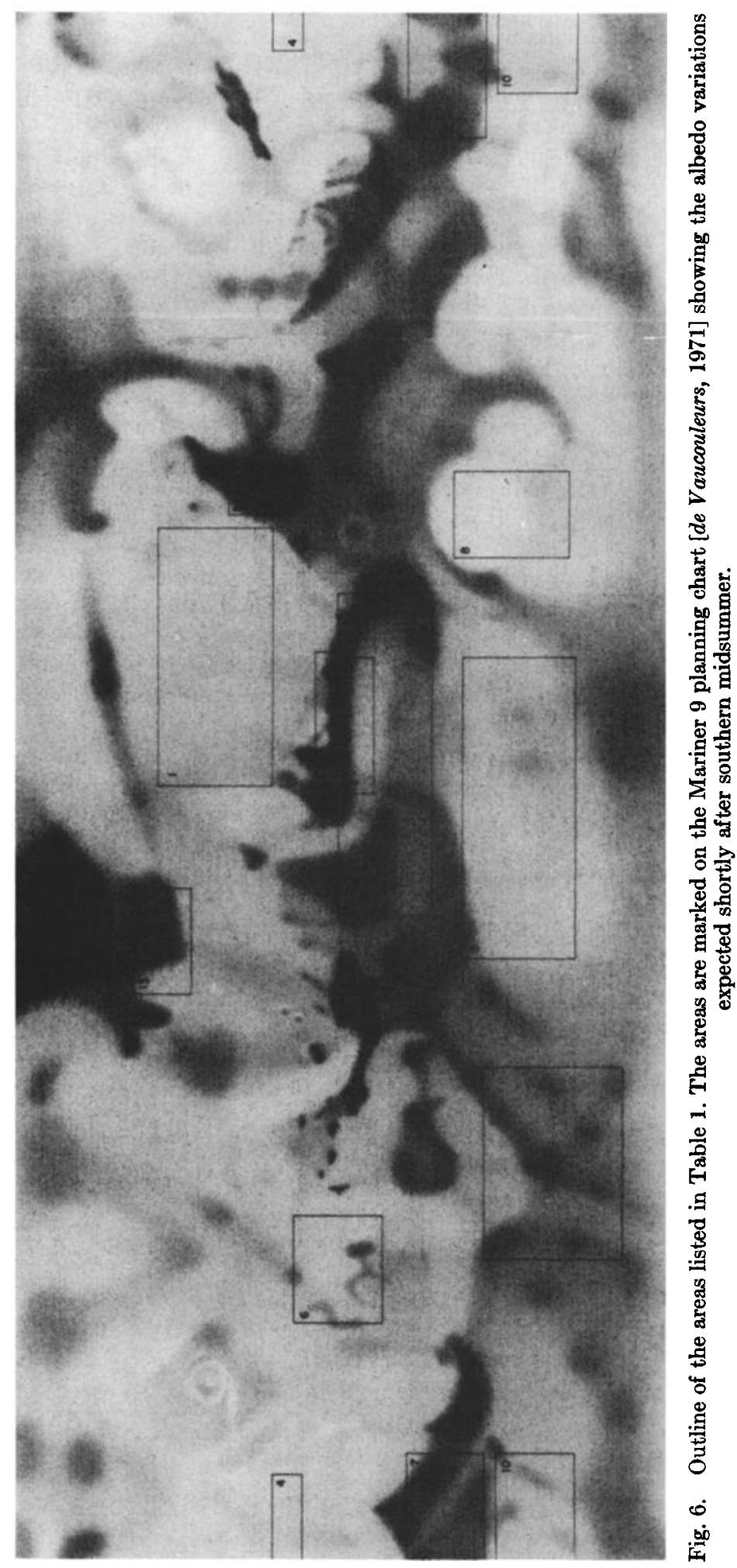


Toward the end of continuous coverage (March 15, 1972) the best-fit inertia approaches $0.007 \pm 0.001$. The albedo was still decreasing steadily, but the asymptotic value must be within $0.25 \pm 0.05$. The large variations in the last 20 revolutions may result from errors in the geometry source tapes. The values of albedo and inertia derived by fitting the 20- $\mu \mathrm{m}$ brightness temperature $T_{\mathbf{w}}$ are similar to those for the $T_{10}$ fit, which is a corollary of the general agreement of $T_{10}$ and $T_{20}$ at equatorial and temperate latitudes. Significant differences between $T_{10}$ and $T_{20}$ were apparent only during early stages of the mission.

It should be interjected that the temperature pattern observed early in the mission can be accounted for by the addition of a single cloud layer to the normal thermal model [Chase et al., 1972; Gierasch and Goody, 1971]. Such a model for the dust-covered planet requires stipulation of at least four additional parameters that vary with latitude and time; these should be contrasted with the three time independent parameters that specify the nominal thermal model. Detailed study of the progression of the dust storm incorporating a dust layer has consequently been omitted from this paper.

The best-fit parameters for the Mariner 69 data, rederived from the brightness temperatures by the same procedure used with the 1971 observations, also are shown in Figure 5. The increased albedo for the Mariner $69 \quad T_{20}$ model fit results from the fact that the average brightness temperatures at $20 \mu \mathrm{m}$ are signifi- cantly colder than those at $10 \mu \mathrm{m}$ for the Mariner 69 data; Neugebauer et al. [1971] took this to indicate an emissivity difference between 10 and $20 \mu \mathrm{m}$. This effect represents a clear difference of about $3^{\circ}$ at $250^{\circ} \mathrm{K}$ between the 1969 and 1971 results. The discrepancy has not been resolved at the present time and may be either in the calibration or a vestigial effect of the dust storm.

\section{Large-Scale Structure}

Several large areas outlined in Figure 6 were selected to look for regional differences in the thermal properties of Mars. The boundaries were chosen to include areas of uniform visual reflectivity on the Mariner 9 planning chart [de Vaucouleurs, 1971] and of uniform general surface morphology as shown on the preliminary Mars chart by Masursky et al. [1972]; polar regions were specifically excluded.

The three large regions near $0^{\circ}$ longitude (1-3 in Table 1) were selected to be representative of classic light and dark areas and to have similar apparent large-scale morphology, in this case fairly uniformly cratered terrain with few volcanic or canyonland features.

Several other regions were selected to cover a range of visual reflectivity, elevation, and surface types. Syrtis Major (5) and Mesogaea (4) are two lightly cratered areas with very different albedos. They are at the same latitude and hence have had nearly identical observational coverage. Hellas (8) is a large area of low elevation (A. B. Binder, private communication, 1971) that has long been associated with white

TABLE 1. Observed Minus Model 10- $\mu \mathrm{m}$ Brightness Temperatures of Selected Areas

\begin{tabular}{|c|c|c|c|c|c|}
\hline & Area & Predawn & Midday & Other & $\begin{array}{c}\text { Residual } \\
\text { Diurnal } \\
\text { Amplitude }\end{array}$ \\
\hline 1. & North light areas & & -8.2 & -19.9 & \\
\hline 2. & Equatorial dark areas & 7.4 & -6.2 & -5.4 & -13.7 \\
\hline 3. & South light areas & 4.8 & -7.3 & -5.4 & -12.1 \\
\hline 4. & Mesogaea & & -13.4 & -22.6 & \\
\hline 5. & Syrtis Major & & -1.5 & & \\
\hline 6. & South Tharsis & -7.2 & -0.1 & -3.7 & 7.1 \\
\hline 7. & Mare Cimmerium & 3.0 & -3.1 & & -6.1 \\
\hline 8. & Hellas & 8.9 & -18.2 & -4.5 & -27.1 \\
\hline 9. & Bosporus region & 2.3 & -7.9 & -3.7 & -10.2 \\
\hline 10. & Electris & 6.1 & -6.6 & -7.2 & -12.7 \\
\hline 11. & Sinus Meridiani & & -3.6 & -10.4 & \\
\hline & Deucalionis Regio & 3.0 & -5.9 & & -8.9 \\
\hline 13. & Niliacus Lacus & & -8.7 & -8.9 & \\
\hline
\end{tabular}


clouds [Slipher, 1964; Smith and Smith, 1972] and is the type example for featureless terrain [Sharp, 1971]. The Bosporos region (9) is at the same latitude and has a visual reflectivity similar to that of the south light area (3) but is less heavily cratered. Mare Cimmerium (7) is a moderately cratered but quite dark area.

One area, south Tharsis (6), was not chosen on the basis of the two maps mentioned above but because, early in the mission, it was noticed as having unusually low predawn temperatures. It is a high-elevation area with low relief except for South Spot volcano and the western extreme of the canyonlands.

In the analysis the temperature data were divided according to local time into predawn, midday (1000-1600), and all other times. As discussed, predawn temperatures are primarily dependent on thermal inertia, and the effect of bolometric albedo is greatest at midday (Figure 4). To avoid the major effects of the dust storm, only observations from the last half of the mission were used. Because of the position of the orbit, essentially no predawn data are available from this part of the mission for areas north of the equator. The differences from the Mariner 69 model are presented in Table 1. It is obvious that the diurnal amplitude of the observed temperatures is generally less than that predicted on the basis of the Mariner 69 data,

The residual temperatures of the three large, heavily cratered areas (1-3) are remarkably similar despite their striking differences in visual albedo. At midday the two light areas were only $1^{\circ}-2^{\circ} \mathrm{K}$ cooler than the equatorial dark area. Likewise, the predawn temperature of the dark area (2) was only $2.5^{\circ} \mathrm{K}$ warmer than that of the south light area (3). The small differences in the amplitude of the diurnal temperature variation thus indicate that there is almost no connection between the classic visual reflectivity and the thermal inertia for these areas. Similarly, the close agreement of the temperature residuals for pairs of areas that have similar surface morphology but generally different visual reflectivities (e.g., areas 3 and $\mathbf{9}$, 7 and 10, and 11 and 12, listed in Table 1) again points to a lack of correlation between the classic brightness and the midday temperatures. In contrast, the areas of Syrtis Major (5) and Mesogaea (4) show approximately the expected correlation between classic brightness and temperature; the $12^{\circ} \mathrm{K}$ difference in the midday temperatures corresponds to a bolometric albedo difference of 0.1 , whereas the visual reflectivity difference is about 0.15 [de Vaucouleurs, 1967]. In general, the midday temperatures appeared to have less dependence on visual reflectivity for cratered areas than for smooth areas. We have in progress a more general study of the relation of surface temperatures to the visual reflectivity as measured from the Mariner 9 television pictures.

There is no a priori way of choosing areas that represent extremes in thermal inertia. Of the 13 areas selected, Hellas has been conspicuous throughout the mission for its small diurnal temperature variations. The amplitude of $68^{\circ} \mathrm{K}$ is $27^{\circ} \mathrm{K}$ less than that predicted by the Mariner 69 model and is the smallest amplitude observed for all these areas; it corresponds to an inertia of about 0.011 . The diurnal amplitude of the south Tharsis region averages $7^{\circ} \mathrm{K}$ greater than the model, by far the largest observed, equivalent to an inertia of about 0.005 .

The visual reflectivity of Mars shows a general dependence on latitude, as is shown in Figure 6. Thus, in addition to the selected areas of Table 1 , the whole planet between $-60^{\circ}$ and $+40^{\circ}$ was divided into latitude bands $5^{\circ}$ wide; temperature residuals from the Mariner 69 model are shown in Figure 7. Surprisingly, the data show a more consistent pattern than the areas selected only on the basis of visual reflectivity do. There are bands with low midday temperatures from $-60^{\circ}$ to $-30^{\circ}$ and from $+10^{\circ}$ to $+20^{\circ}$ latitude. Although the variations are small, the trends are significant in view of the large number of observations incorporated in the data.

Predawn observations. In addition to selecting areas on the basis of predicted or observed physical properties, a large group of areas was studied on the basis of their being observed after midnight local time and before sunrise. For these areas the surface temperatures depend primarily on the thermal inertia, since the effects of albedo variations and slopes are then the smallest (see Figures 4 and 8). The observed predawn $10-\mu \mathrm{m}$ brightness temperatures are shown in Figure 9 as differences from the Mariner 69 model. Because of physical limits in 


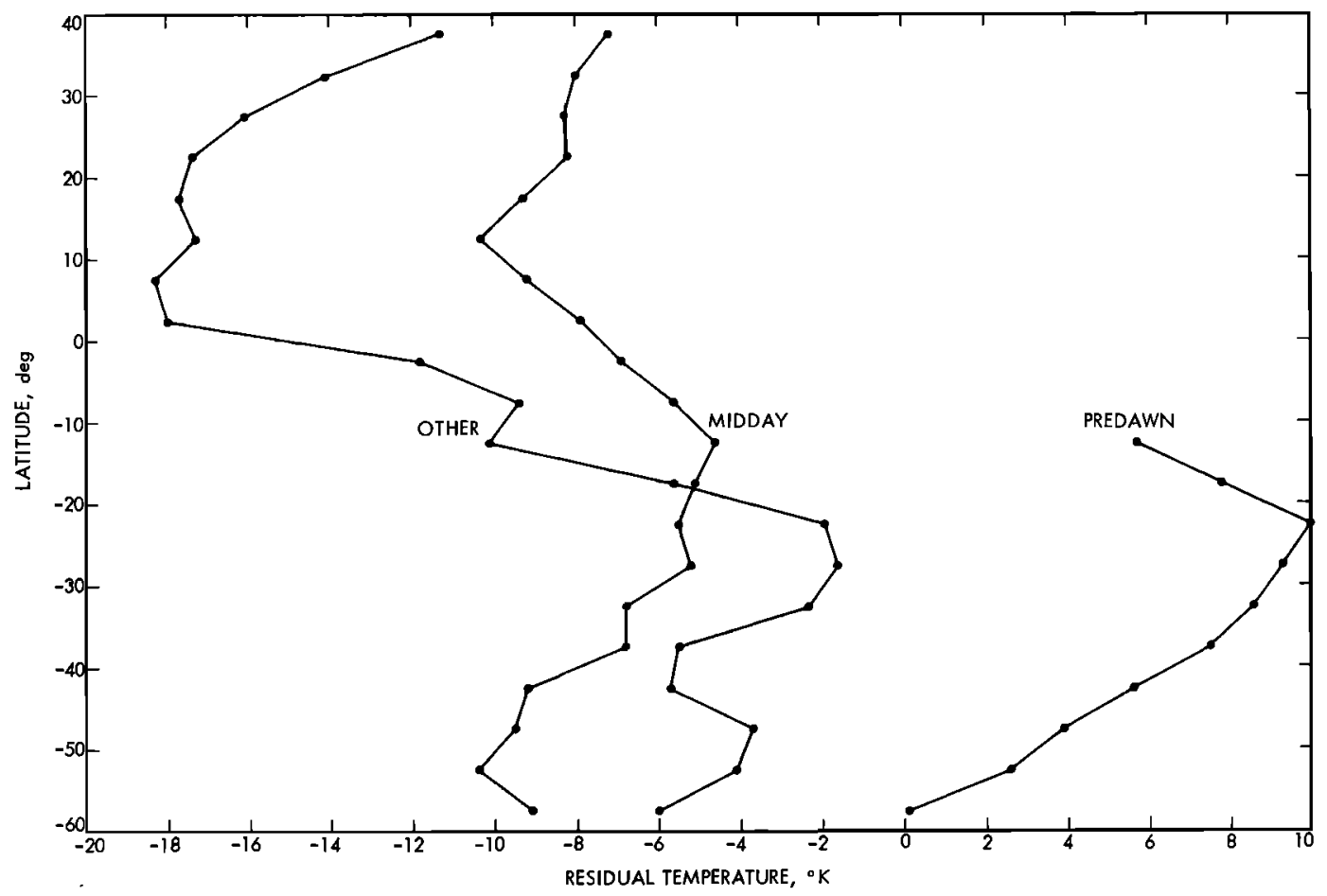

Fig. 7. Average temperature differences, observed minus model, divided according to local time as a function of latitude from January through March 1972. The variation of the midday temperature average generally follows the albedo pattern depicted by earth-based observers.

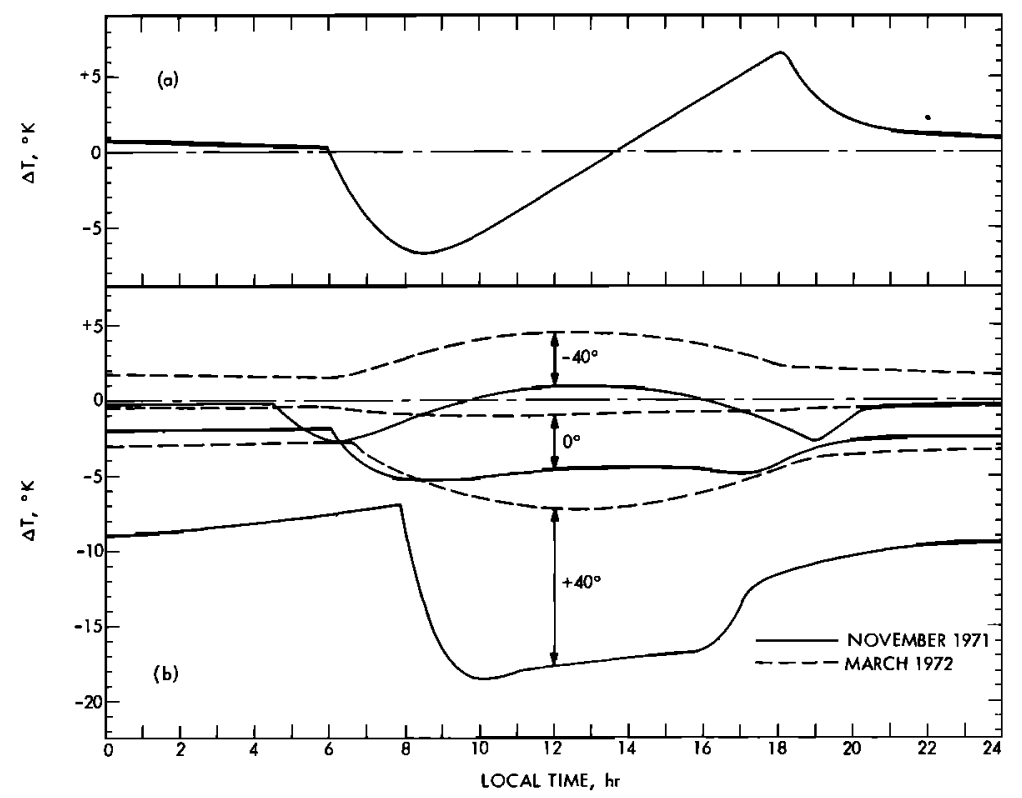

Fig. 8 (a) The temperature change attributable to a $5^{\circ}$ west-facing slope as a function of Mars local time. Variation of this function with latitude and season is small and has been omitted from the figure. (b) The temperature change attributable to a $5^{\circ}$ north-facing slope as a function of Mars local time for three latitudes $\left(0^{\circ}, \pm 40^{\circ}\right)$. Solid lines depict this dependence shortly after orbit insertion; dashed lines represent the situation near the end of the standard mission. 

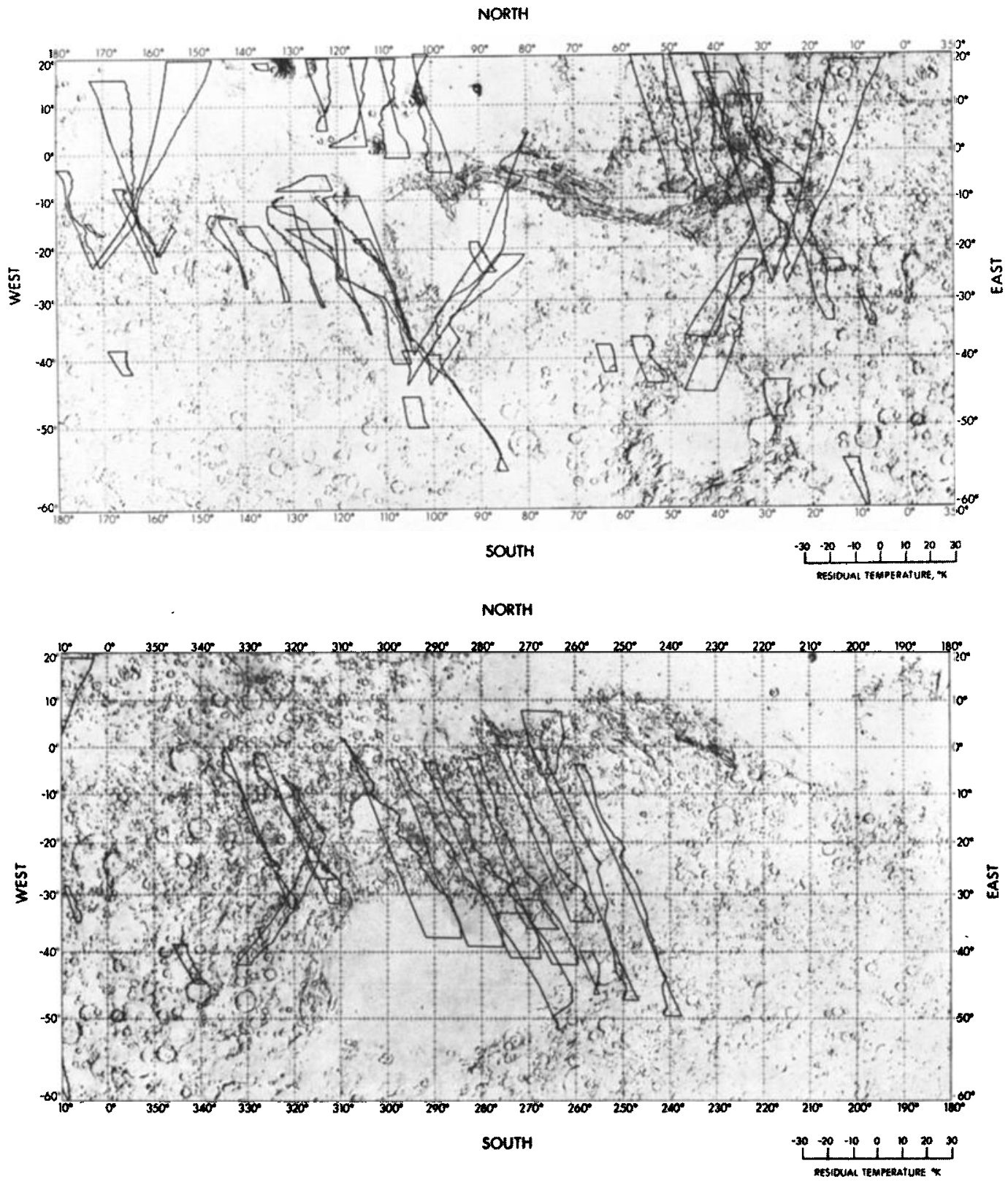

Fig. 9a.

Fig. 9. Predawn residual temperatures. The observed temperature minus the Mariner 69 model temperature is shown as an offset from the trace of the radiometer observation across the planet. Temperature increases to the right as indicated in the legend; no offset represents a $+10^{\circ}$ difference. (a) Data from December 14, 1971, to January 13, 1972. (b) Data from January 14 to February 12, 1972. (c) Data from February 13 to March 13, 1972.

viewing directions, there is little predawn coverage north of the equator, and the northern limit moved south through the mission.

Many pronounced local and regional varia- tions were observed in predawn temperatures. Early in the mission these variations were partly masked by the dusty atmosphere. On the basis of earth-based measurements, Morri- 

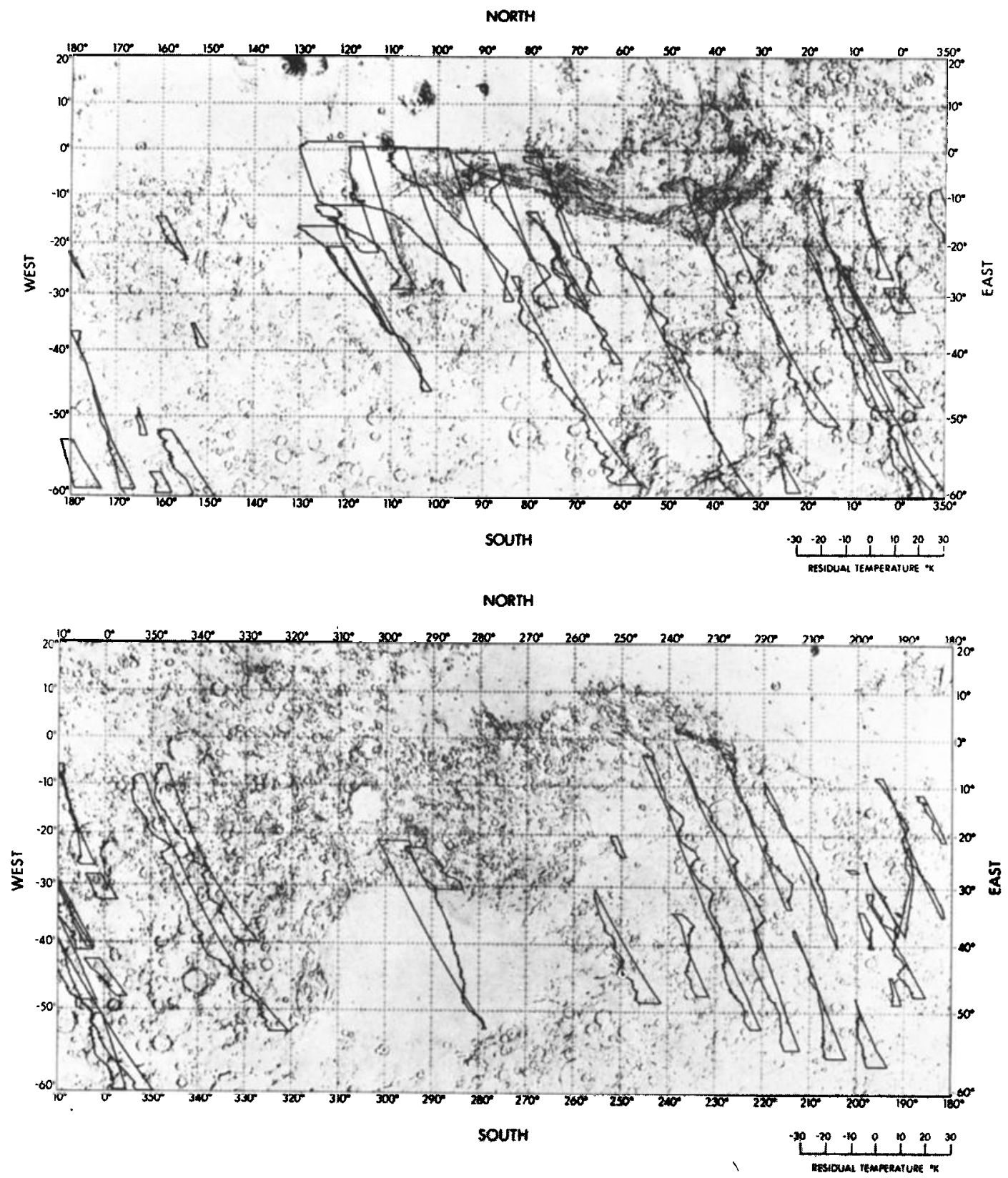

Fig. $9 b$.

son et al. [1969] have suggested that lower visual reflectivity is correlated with higher thermal inertia. Although both lower albedo and higher thermal inertia increase predawn temperature, the temperatures mensured from Mariner 9 do not consistently increase onto dark areas. Although some examples of such correla- tion can be found (e.g., between Mare Tyrrhenum and Ausonia (latitude $-30^{\circ}$, longitude $\left.250^{\circ} \mathrm{W}\right)$ ), many counter examples exist (e.g., Thaumasis and Bosporos $\left.\left(-36^{\circ}, 72^{\circ} \mathrm{W}\right)\right)$; some of the highest-contrast borders show little effect at all (e.g., Sinus Sabaeus and Deucalionis Regio $\left(-10^{\circ}, 340^{\circ} \mathrm{W}\right)$ and the borders 

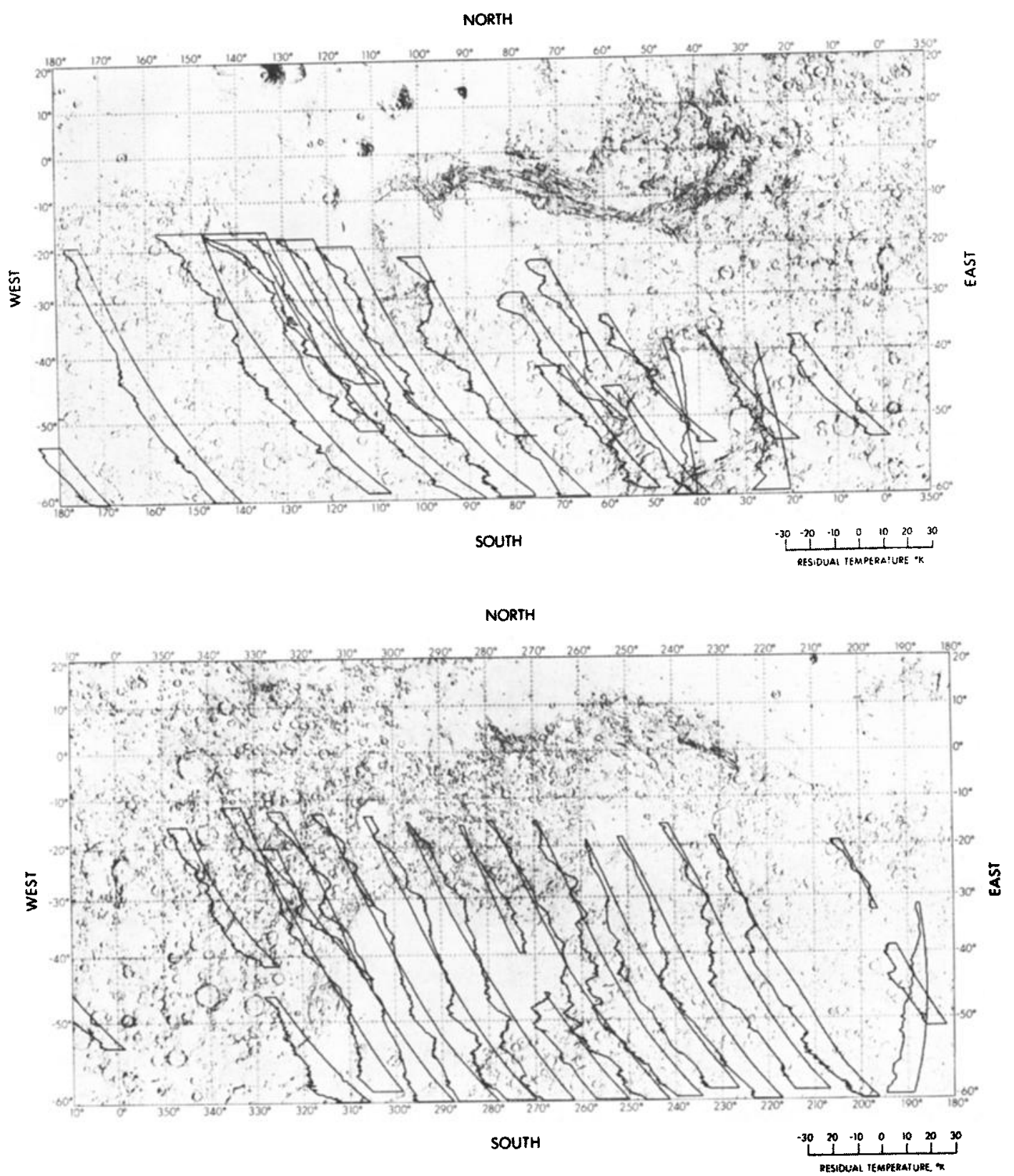

Fig. 9c.

of Hellas). The range of predawn temperatures observed is far greater than could be attributed to albedo variations and implies major variations of the thermal inertia.

Using the preliminary Mars chart of Masursky et al. [1972], no consistent relation has been discovered between the predawn tempera- tures and morphology. However, a cautionary note on the preliminary Mars chart states that positional inaccuracies as large as $60 \mathrm{~km}$ exist throughout the chart. Such a positional error is greater than the field of view for the radiometer during predawn observations. Some crater rims appear as local temperature increases (e.g., 
$\left.-57^{\circ}, 20^{\circ} \mathrm{W}\right)$, although counter examples are common $\left(-17^{\circ}, 300^{\circ} \mathrm{W}\right)$. The parallel large canyons, but not the cross-patterned area at their western extreme (the chandelier), appear warmer than the surrounding areas before sunrise.

Many of the features seen in the predawn data are as small as the field of view of the radiometer, approximately $40 \mathrm{~km}$ at this part of the orbit. The large number of these features that are at the scale of the radiometer's resolution suggests that the real sources are in fact smaller and hotter than indicated by the apparent brightness temperature.

A few of the more pronounced local predawn temperatures that are high were also handplotted on the Mariner 9 television pictures. The pictures were, of necessity, taken at other times; thus small inconsistencies in relative location may exist. No general rule relating predawn temperatures to specific morphologies has become apparent. There are examples of local temperature increases corresponding to small dark areas or to areas near the borders of rough geologic provinces and examples where there is no apparent reflectivity or topographic feature at the location of the thermal contrast.

A major feature of the predawn observations is the large region of very low temperatures around Tharsis. As this part of Mars was known to be high, a general correlation between temperature residuals and altitude was sought. The predawn temperatures and elevation data were both averaged over $5^{\circ}$ square areas from $-30^{\circ}$ to $+30^{\circ}$ latitude. Two sets of elevation data were used: (1) a topographic map based on data from all available sources (A. B. Binder, private communication, 1971) and (2) elevations derived from the 1971 Haystack radar observations (G. H. Pettengill, private communication, 1972). When either set of elevation data is used, there is a general decrease of average predawn temperature with increasing height of about $-1.5^{\circ} \mathrm{K} / \mathrm{km}$, although the data show wide variations. Because thermal conductivity of granulated rock depends on gas pressure when the mean free path of the gas molecules is comparable to the soil pore dimension, a decrease in thermal inertia with height is expected; the mean free path is about $10^{-3} \mathrm{~cm}$ at the mean surface pressure and temperature on Mars. Wechsler and Glaser [1965] have compiled thermal conductivity measurements of granulated samples made over a range of pressures. For pressures between 1 and $10 \mathrm{mb}$, the thermal conductivity of a wide range of powdered materials increases approximately as the square root of the gas pressure, or the thermal inertia increases as the fourth root of the pressure. For a height range of $\pm 6 \mathrm{~km}$ around the mean surface level, corresponding to $\pm 3 / 4$ of the Martian scale height, this corresponds to a $\mp 20 \%$ change in thermal inertia or a $\mp 4.2^{\circ} \mathrm{K}$ change in the predawn temperatures. Thus decreasing gas pressure can account for an elevation dependence of about $-0.7^{\circ} \mathrm{K} / \mathrm{km}$. This is about half that observed, and the remaining amount is attributed to a decrease of average thermal inertia with increasing elevation.

G. H. Pettengill (private communication, 1972) has used the 1971 Haystack radar observations to derive the dielectric constant and root mean square slopes of a band of the Martian surface overlapped by the predawn data. Although the dielectric constant increases with density and the amount of solid rock [Campbell and Ulrichs, 1969] and might be expected to show a correlation with thermal inertia, as has been observed for the moon [Zisk et al., 1971], no significant correlation was found between predawn temperatures and the data presented by Pettengill.

Implied physical properties. The predawn data are the most directly interpretable of the thermal data presented here. Figure 10 shows the relation between the thermal inertia and the diurnal amplitude and predawn temperatures. The diurnal amplitude, where determined, is a somewhat better indicator of thermal inertia than the predawn temperature, as it has a smaller dependence on albedo.

As discussed by Neugebauer et al. [1971], the main physical property determining thermal inertia for materials relevant to Mars is thermal conductivity, which is closely related to particle size. Wechsler and Glaser [1965, Figures 4, 8, and 11] have compiled, at several gas pressures, thermal conductivity measurements of powdered rocks with particle diameters from 0.004 to $0.08 \mathrm{~cm}$ and have interpolated them across the pressure range relevant to Mars. Over this particle size range, the thermal conductivity varies approximately linearly with 


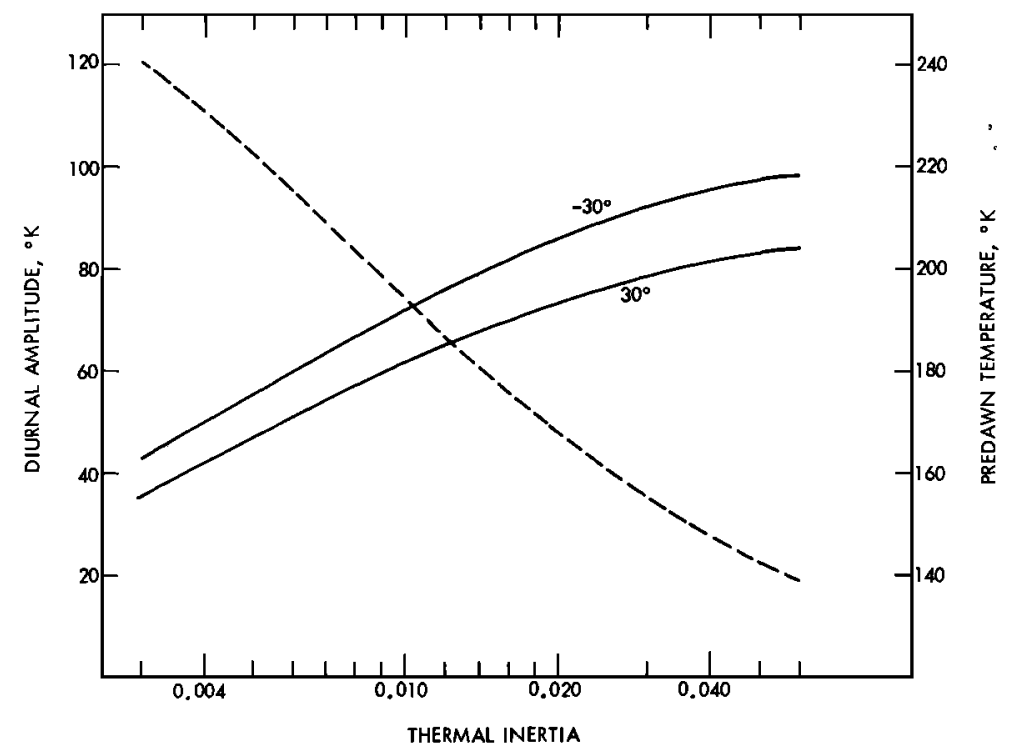

Fig. 10. Diurnal temperature amplitude at $-30^{\circ}$ latitude (dashed line) and predawn temperatures at $+30^{\circ}$ and $-30^{\circ}$ latitude (solid curves) for a constant albedo of 0.3 during January 27, 1972. Although the absolute predawn temperature depends on latitude and Martian season, the shape of the curve, and hence the residual temperature, is almost independent of season or latitude away from the polar regions.

particle size. We are unaware of any thermal conductivity measurements at relevant pressures for geologic materials with mean particle diameters between $0.1 \mathrm{~cm}$ and several centimeters. Since measurements for centimetersized particles are lacking, a smooth curve drawn through the available data for small particles and asymptotically approaching the conductivity of solid rocks at $100 \mathrm{~cm}$ has been adopted (Figure 11). This is an estimate of the thermal conductivity of uniformly sized lithic soils at $6 \mathrm{mb}$ but should serve as an indication of the surface properties required by the observed temperatures.

At the scale of classic features, the extremes of predawn residual temperatures and diurnal amplitudes listed in Table 1 imply a range of inertia from 0.005 for south Tharsis to 0.012 for Hellas. Interpreted as regional variations of uniformly sized soil, the mean particle size ranges from 0.015 to $0.2 \mathrm{~cm}$. The fact that these two areas also appear to represent the elevation extremes on Mars suggests a general dependence of temperature variations on height. A possible cause is the size-sorting nature of eolian transport and the inability of the Martian atmosphere to carry la rger material to high elevations.
The derivation of surface physical properties within the Hellas basin is complicated by the apparent presence of an exceptionally persistent local dust storm. Examination of television pictures taken late in the mission indicates that the surface was obscured through most of the mission. The apparent thermal inertia would be significantly affected if the dust caused strong radiative coupling through more than the lower few hundred meters of the atmosphere.

At the limit of the radiometer's spatial resolution, the lowest residual predawn temperature found, $-15^{\circ} \mathrm{K}$ in the Tharsis area, corresponds to an inertia of 0.0035 and a nominal particle size of $0.006 \mathrm{~cm}$. There cannot be any appreciable fraction of material greater than $0.02 \mathrm{~cm}$ in diameter exposed on the surface in that area. The corrections for the lower gas pressure and conductive coupling to the atmosphere are of opposite sign, and a nominal net correction would result in approximately a $20 \%$ increase in these particle sizes. Because the expected effect of the dust storm is to increase the apparent inertia, these estimates of minimum particle size are likely to fall within the actual range. Estimates of maximum particle size could be moved to unrealistic values by the effects of a dusty 


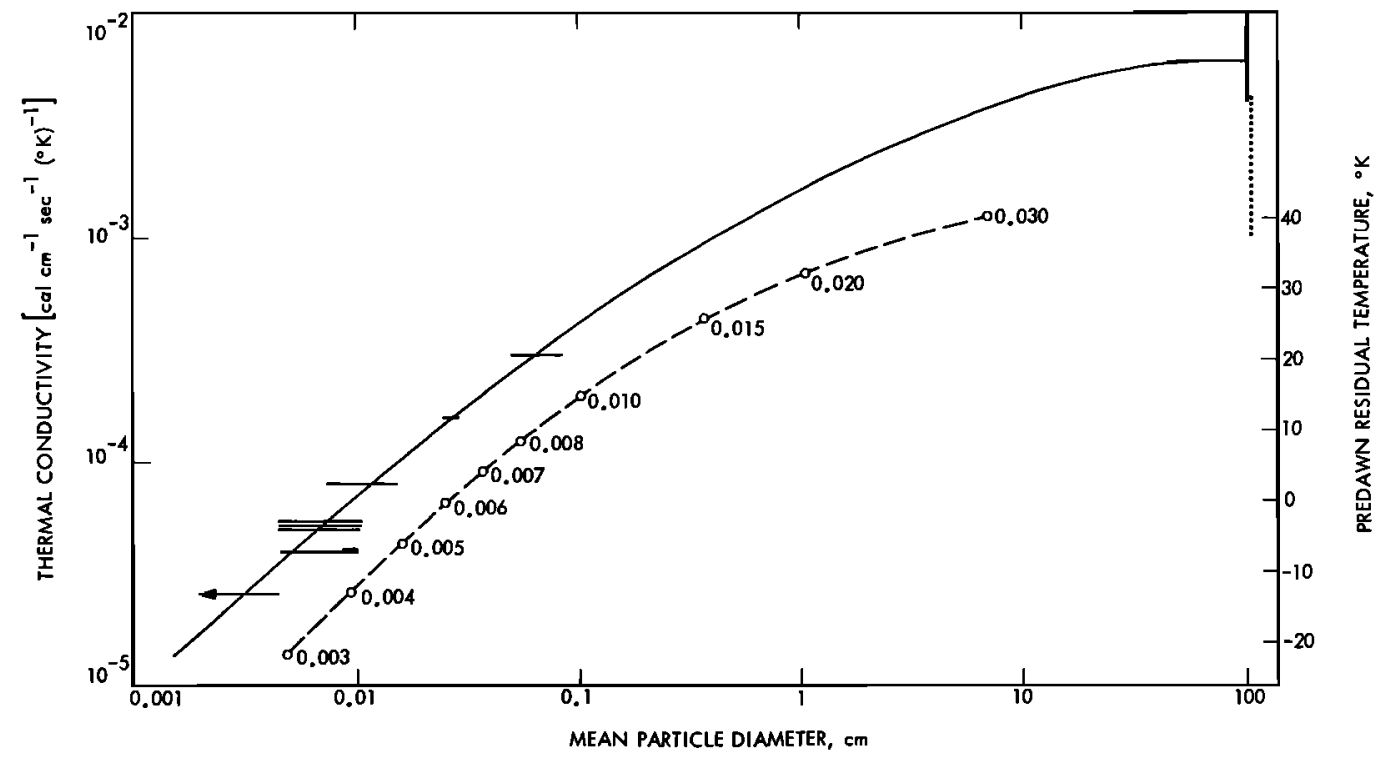

Fig. 11. Nominal dependence of thermal conductivity and inertia on particle size. The data are taken from Wechsler and Glaser [1965]. The solid line is a smooth curve handplotted between the data for fine particulate samples and solid rocks. The horizontal lines represent reported size ranges. The vertical solid line is the range of conductivities for dense igneous rocks, whereas the vertical dotted line represents vesicular basalts. The dashed line represents the computed relation between the particle size and the predawn residual temperature (i.e., observed temperature minus Mariner 69 model temperature) as presented in Figure 9. The calculations assume a volume specific heat $\rho c$ of 0.24 ; calculations are for $-30^{\circ}$ latitude on January 28, 1972 , but are not a strong function of latitude, date, or time before sunrise.

atmosphere; hence only data from the last half of the mission are considered. In contrast to the apparent localization of the lowest inferred inertias around south Tharsis, high predawn residual temperatures of about $+20^{\circ} \mathrm{K}$ were found at several locations. The highest predawn residual temperature found after January 15, 1972, was $+23^{\circ} \mathrm{K}$ measured near the north edge of Hellas, outside the area of the local dust storm mentioned above, corresponding to an inertia of about 0.017 and a mean particle size of about $0.5 \mathrm{~cm}$. It should be cautioned that the thermal model used thus far is physically naive. Of the various complications possible, the occurrence of nonuniformly sized material and thermal coupling with the atmosphere probably are dominant.

As an example of nonuniformly sized material, we consider the occurrence of large blocky material with a high inertia exposed on a surface whose inertia is small. This requires assignment of the inertias of both the homogeneous surface and the blocky material. As an example, if blocks $10 \mathrm{~cm}$ or larger with an inertia 0.030 are exposed on a surface of 0.01 cm particles with an inertia 0.004 , the observed temperatures listed in Table 1 imply that the fraction of the surface covered by exposed blocks ranges from 5 to $35 \%$. The small diurnal amplitude observed locally near Hellas would require $70 \%$ exposure of blocky material.

Conductive heat transfer between the atmosphere and the surface tends to decrease the diurnal temperature variation in comparison with what would result if the atmospheric boundary layer were perfectly insulating. The inertia indicated by the daily temperature variation is therefore larger than should be attributed to the surface material alone. Based on calculations of Gierasch and Goody [1968], Neugebauer et al. [1971] estimated that this process increases the apparent inertia by about $10 \%$, but there is a large uncertainty associated with this estimate because of our lack of knowledge of the boundary layer conditions on Mars. During the dust storm, thermal coupling between 
KiefFer ET AL.: MARINER 9 Mission

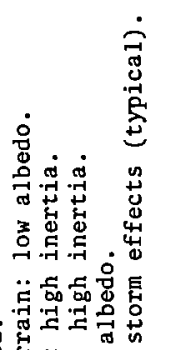

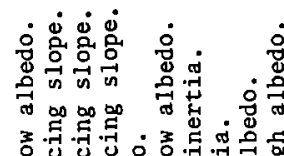

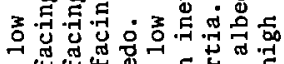

की

$\square$

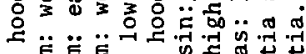

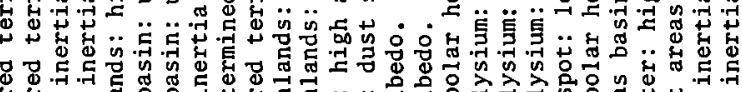

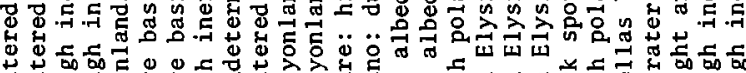

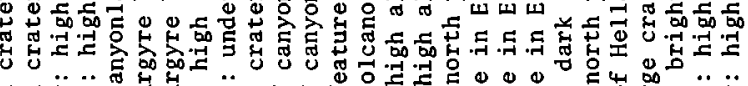

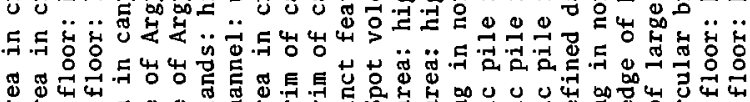

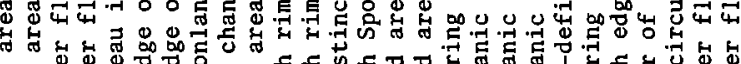

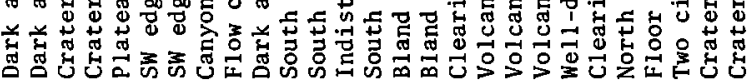
NTNNN

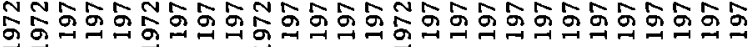

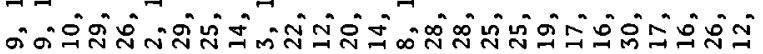

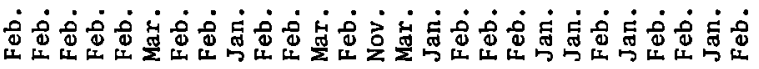

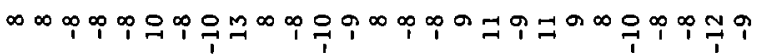

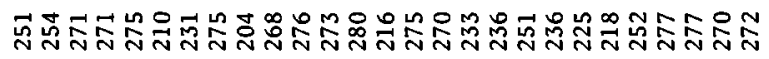

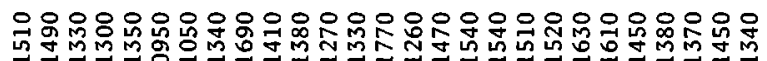

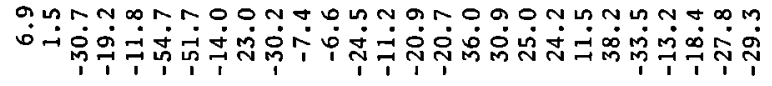
苟

ๆ

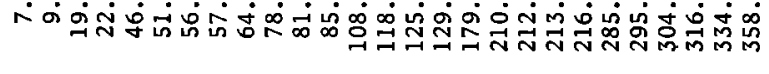




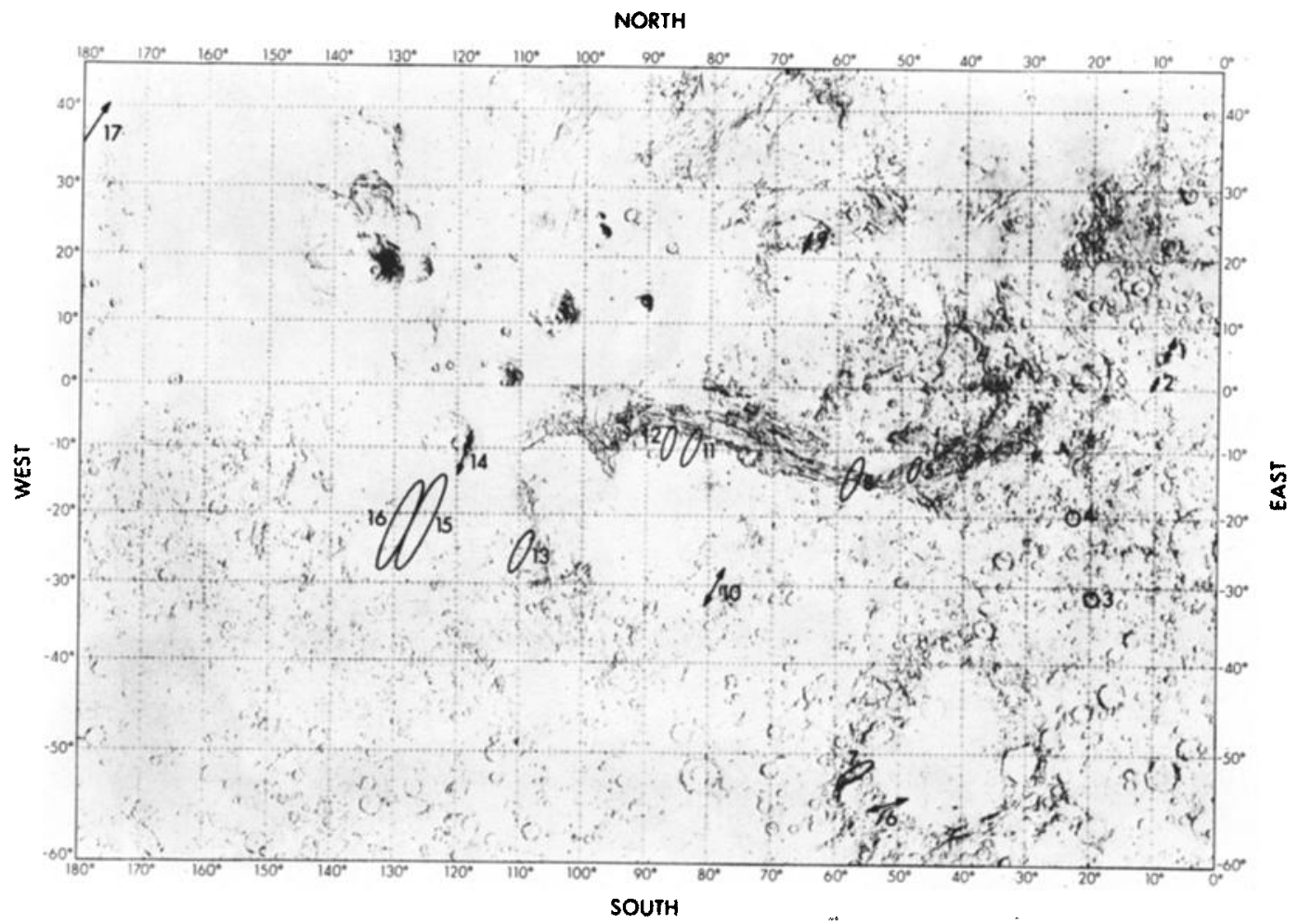

Fig. $12 a$.

Fig. 12. (a) Locations and approximate extent of the localized thermal features listed in Table 2. The 16 areas that are colder than their backgrounds are shown by ellipses, and the 11 warmer areas are shown by double-pointed arrows. Longitudes $0^{\circ}$ to $180^{\circ} \mathrm{W}$. (b) Same as (a). Longitudes $180^{\circ}$ to $360^{\circ} \mathrm{W}$.

the surface and the atmosphere almost certainly increased over that estimated for clear conditions. Such effects have not been accounted for in this analysis; consequently, the derived thermal inertia presented here must be somewhat larger than the actual values for the surface material.

\section{Localized Thermal Features}

The search for localized thermal features was one of the primary goals of the infrared radiometer experiment. A preliminary search has been made for local deviations from the mean thermal background that can be associated with features observed by the television cameras. The method of selecting the local anomalies has eliminated all areas larger than about 800 km (about 15 areocentric degrees). The search was systematically conducted for all revolutions between January 3,1972 , when the television mapping sequence started and it was first possible to easily identify surface features, and March 13, 1972, when the signal-to-noise ratio became significantly degraded. Data taken when the instrument platform was in motion have been omitted. Several hundred areas between latitudes $-60^{\circ}$ and $+40^{\circ}$ on the planet appear to be localized thermal features differing in temperature from their surroundings by $4^{\circ} \mathrm{K}$ or more. Of these, the 27 tabulated in Table 2 show differences of at least $8^{\circ} \mathrm{K}$; their locations and approximate extents also have been plotted on a television mosaic of the planet in Figure 12, where it can be seen that many of the areas may be associated with topographic features on the planet.

In Table 2 , an attempt has been made to delineate the probable cause of the temperature variations on the basis of their visual appearance. Thus, for example, the Martian 


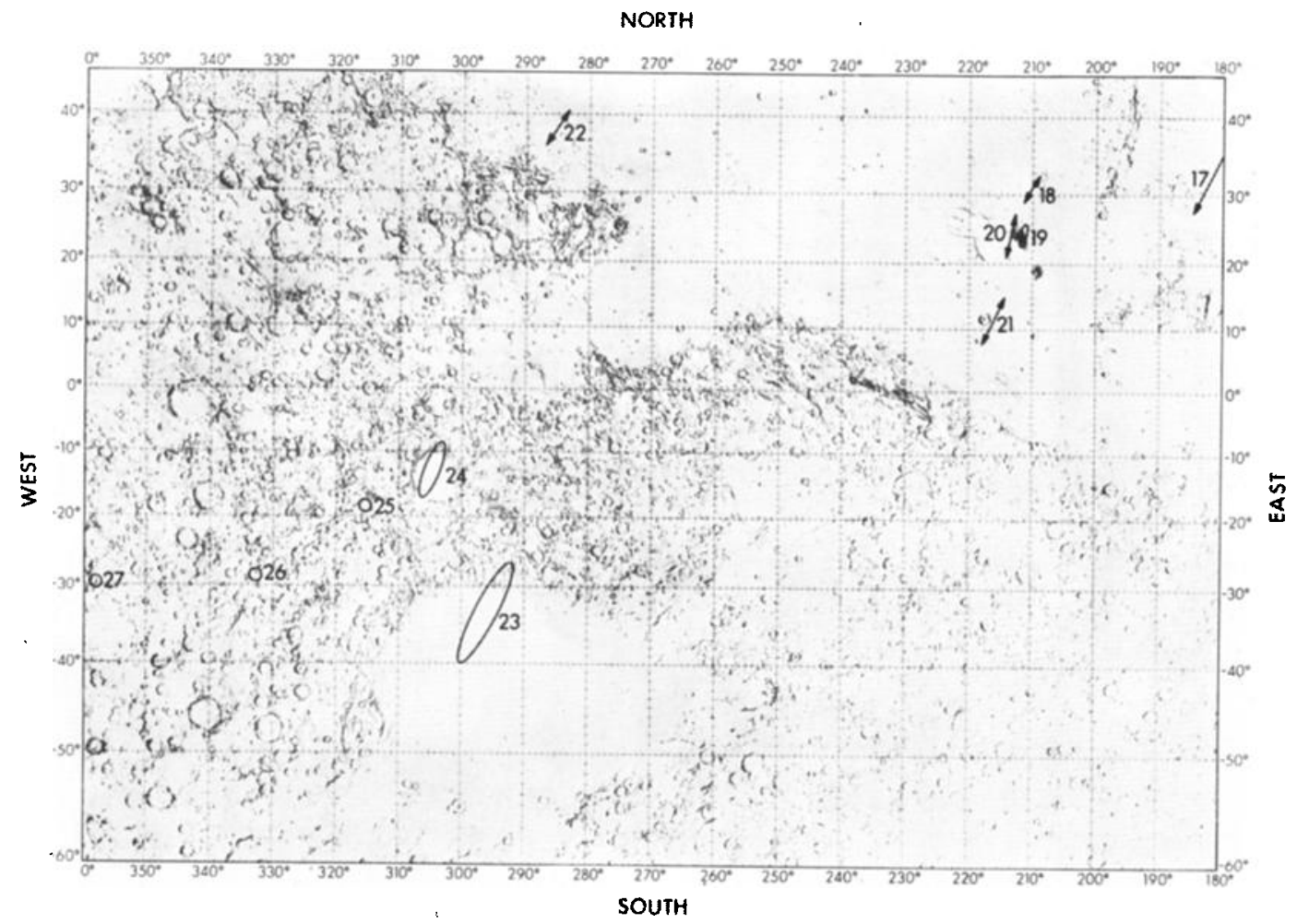

Fig. $12 b$.

canyonlands (areas 5, 8,11, and 12), characterized by sharp topographic boundaries, may be areas of distinct thermal inertia. The large crater at $-13^{\circ}$ latitude and $305^{\circ}$ longitude may be another such area (24). One clear example of a thermal feature probably caused by an albedo variation is the dark area (21) near the southern boundary of Elysium at $+11.5^{\circ}$ latitude and $216^{\circ} \mathrm{W}$ longitude. This area also has been found by the radiometer onboard the Russian Mars 2 spacecraft to be anomalously warm. In general, an examination of the uncorrected television pictures shows a correlation between temperatures and visual albedo on a small scale.

Local temperature features also may be caused by topographic slopes. To first order, those thermal effects caused by topographic slopes may be approximated by assuming that they correspond to an effective translation in latitude, longitude, or a combination of the two, as demonstrated in Figure 8. Although large slopes might be expected in the canyonlands area $(5,8,11$, and 12$)$, their effect on tem- perature should be negligible at the times indicated for these anomalies. Slopes of the order of $10^{\circ}$ would account for the thermal features observed in areas 19 and 20 , on the flanks of a volcanic mountain in Elysium.

The inverse of the procedure in the preceding paragraph has been applied to a slope analysis of the volcanic mountain called South Spot. By chance, one radiometer scan passed in an approximate east-west direction directly over the central crater. If it is assumed that albedo and thermal inertia are uniform across the volcano and that the floor of the central crater is locally flat, temperature differences from a uniform background may be interpreted as local slopes. Integration of these slopes along the path of the scan yields the profile shown in Figure 13. The results are in fair agreement with altitude profiles obtained by the ultraviolet spectrometer and infrared interferometer spectrometer experiments [A. L. Lane, personal communication, 1972; Conrath et al., 1973].

Several cool areas observed by the radiometer during daylight hours have been identified in 


\section{MARS COOROIMATES}
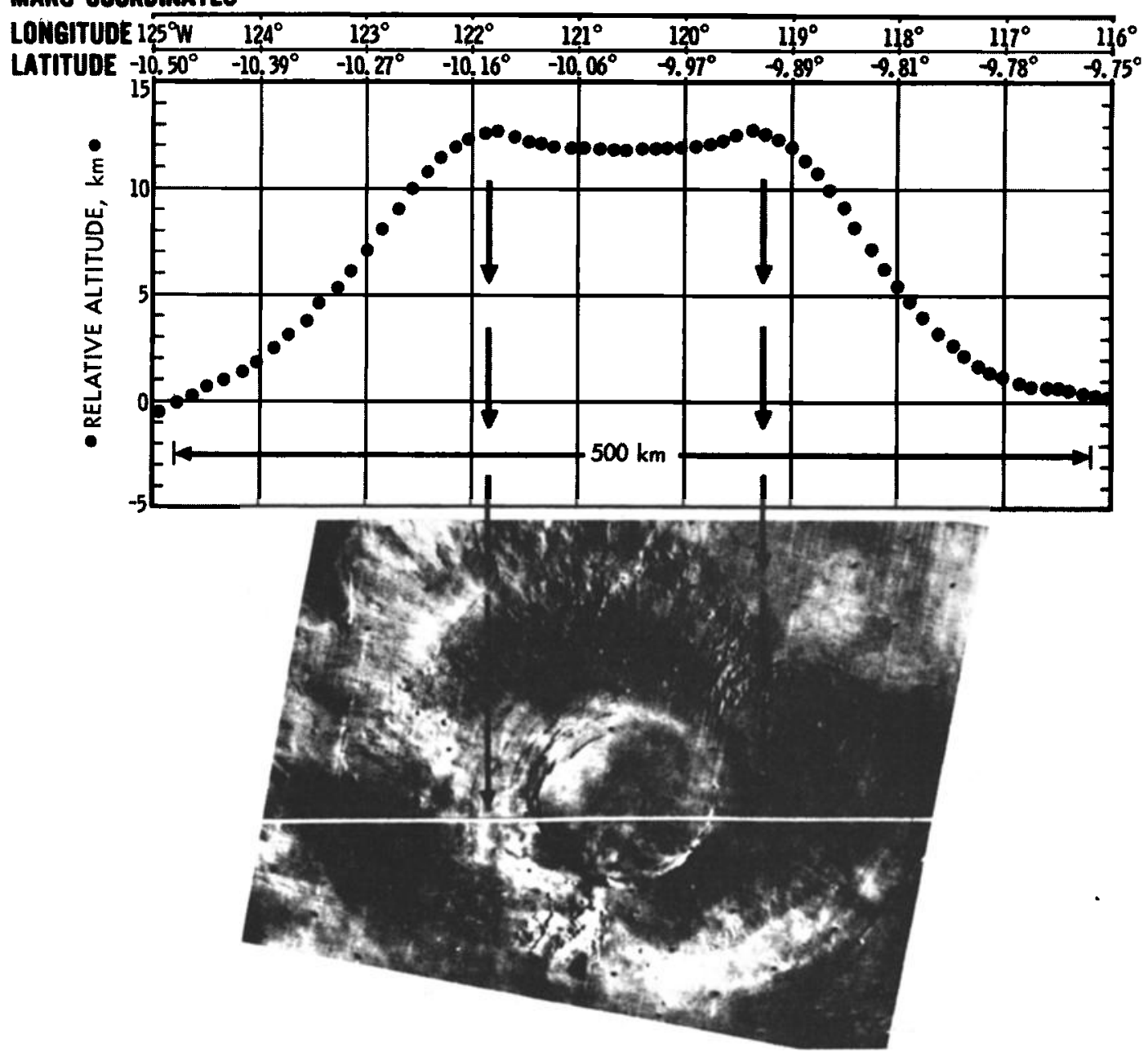

Fig. 13. Altitude profile of South Spot (Nodus Gordii, $-9.5^{\circ}, 120^{\circ} \mathrm{W}$ ). Vertical scale may be in error by as much as $\pm 50 \%$ because of simplifying assumptions.

the television images as clouds [Leovy et al., 1973]. None of these apparent clouds caused temperature drops as large as $8^{\circ} \mathrm{K}$. Inhomogeneities in extensive cloud cover may produce apparent warm areas; areas 17 and 22 near the southern edge of the north polar hood provide examples tentatively identified with holes in clouds.

Four cold anomalies (areas 3, 4, 26, and 27) are associated with impact craters that appear in the much-enhanced television images to have bright areas on their floors. From their appearances, one might be tempted to identify them as water frost deposits on the crater floors. Since these observations were obtained during the warmest part of the day, however, it is unlikely that they represent frost deposits, and the temperature drop probably is due to the albedo or inertia variation. The albedo variation necessary to explain the observed temperature $(\Delta A \cong 0.1)$ is larger than the apparent reflectivity difference in the television images. Therefore these areas may also have higher inertia than the Martian average.

One of the objectives of the infrared radiometer experiment was to search for possible sites of active volcanism. No areas of Mars as large as the projected radiometer field have been observed with brightness temperatures in excess of $300^{\circ} \mathrm{K}$. If a hot spot is smaller than the 
projected area of the radiometer field, the observed brightness temperature will clearly be less than the actual temperature of the feature. A temperature deviation of at least $2^{\circ} \mathrm{K}$ is required for certain recognition. In Figure 14 the detectability of features as a function of their linear size and inherent temperature is indicated for the $10-\mu \mathrm{m}$ channel of the radiometer; the background is taken to be $250^{\circ} \mathrm{K}$, and the hot spot is presumed centered in the field of view. The warm anomalies listed in Table 2 would have to fall in the shaded area of Figure 14 to be attributed to internal heat. An upper limit to expected temperature of an active lava lake is near $1500^{\circ} \mathrm{K}$. No such hot spot greater than $0.5 \mathrm{~km}$ has been observed from distances of $2000 \mathrm{~km}$ or less. A lava lake would have to be at least $0.2 \mathrm{~km}$ in extent and be within the radiometer's field of view to be detected as a $3^{\circ} \mathrm{K}$ rise. Even if Mars had twice the volcanic activity of earth, the probability of observing such a feature would be very small.
Several thermal features were observed at times of day when they could not readily be attributed to inertia variations, and no appropriate reflectivity or slope variations were evident. Although these currently have no probable cause identified, they may be due to some unusual combination of surface properties, and there is no other evidence to suggest that they are due to internal heat flow. Further investigation of these features is in progress.

\section{SUMMARY}

The results of the preliminary analysis of the Mariner 9 radiometer data can be summarized as follows.

Once the planetwide dust storm had subsided, the gross thermal characteristics of Mars agreed qualitatively with those observed with a poorer spatial resolution in 1969 . The ranges in inertia and albedo, $0.004 \lesssim I \lesssim 0.017$ and $0.2 \lesssim A \lesssim$ 0.4 , respectively, were comparable for both

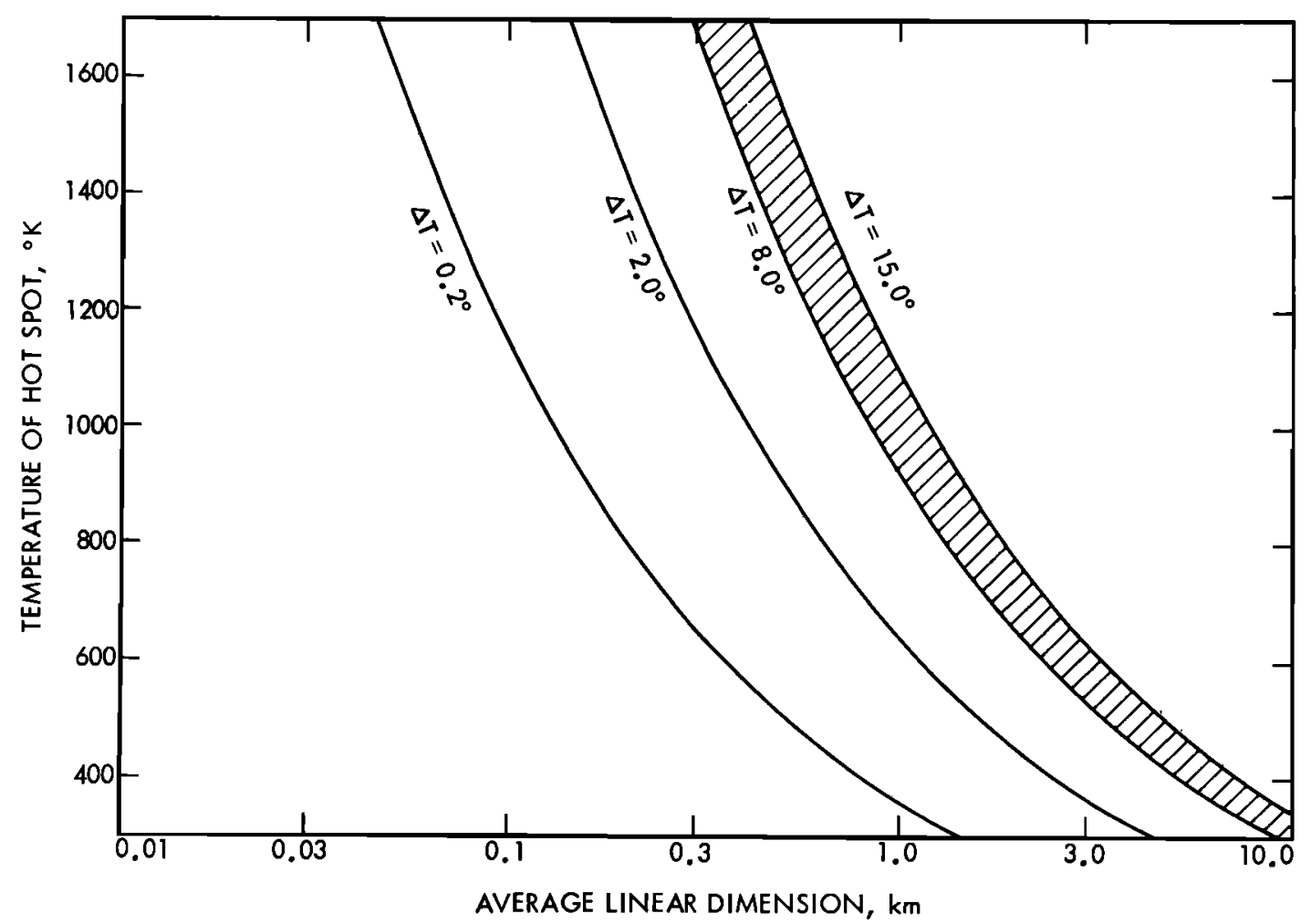

Fig. 14. Detectability of a hot spot smaller in extent than the radiometer field of view as a function of its average linear dimension. The digitization limit is represented by the $0.2^{\circ} \mathrm{K}$ curve. The warm anomalies listed in Table 2 would have to be found in the shaded area to be attributable to internal heat. 
missions. Again, thermal features were visible at the limit of the spatial resolution.

The dust storm changed the thermal pictures of Mars radically. At the observed height of the storm the average dayside temperatures of the planet were lower by about $20^{\circ} \mathrm{K}$ than those without the dust storm.

Over large-scale physiographic visual features, no univocal correlation between albedo and thermal inertia seems to exist. In the small scale, fluctuations in thermal inertia apparently unrelated to albedo changes appear in predawn data. Small-scale brightness variations visible in television pictures generally are associated with simultaneous temperature fluctuations of approximately the expected amplitude, but this is understood as a consequence of the daytime dependence of temperature on albedo, which is stronger than its dependence on thermal inertia. In general, it appears as if albedo and thermal inertia are not dependent on only one and the same property of the Martian soil.

At the limit of resolution of the radiometer, there are many areas that differ significantly from their surroundings. These differences, however, are plausibly explained in terms of modest variations in thermal inertia, bolometric albedo, and topographic slopes without having to invoke internal heat sources.

The surface particle sizes derived from the observed thermal inertias vary between 0.006 and $0.5 \mathrm{~cm}$, a range wider than that previously estimated.

Acknowledgments. We thank all the members of the Mariner 9 project for their help with this experiment; it truly was theirs. We especially thank Ms. J. Bennett, A. Law, and R. Mehlman, who were instrumental in decreasing the confusion of receiving such a large volume of data, and Ms. S. Higley and Ms. E. van der Wyk for their help in preparing this manuscript.

This paper presents the results of one phase of research carried out at the Jet Propulsion Laboratory, California Institute of Technology, under contract NAS 7-100, sponsored by the National Aeronautics and Space Administration.

\section{REFERENCES}

Campbell, M. J., and J. Ulrichs, Electrical properties of rocks and their significance for lunar radar observations, J. Geophys. Res., 74, 5867, 1969.

Chase, S. C., Jr., Infrared radiometer for the 1969
Mariner mission to Mars, Appl. Opt., 8, 639, 1969.

Chase, S. C., Jr., E. Miner, G. Münch, and G. Neugebauer, Infrared radiometry experiment for Mariner Mars 1971, Icarus 12, 46, 1970.

Chase, S. C., Jr., H. Hatzenbeler, H. H. Kieffer, E. Miner, G. Münch, and G. Neugebauer, Infrared radiometry experiment on Mariner 9, Science, 175, 308, 1972.

Conrath, B., R. Curran, R. Hanel, V. Kunde, W. Maguire, J. Pearl, J. Pirraglia, J. Welker, and T. Burke, Atmospheric and surface properties of Mars obtained by infrared spectroscopy on Mariner 9, J. Geophys. Res., 78, this issue, 1973.

de Vaucouleurs, G., A low-resolution photometric map of Mars, Icarus, \%, 310, 1967.

de Vaucouleurs, G., A Mars chart for the Mariner flights, Sky and Telescope, 41, 283, 1971.

Gierasch, P. J., and R. M. Goody, A study of the thermal and dynamical structure of the Martian lower atmosphere, Planet. Space Sci., $16,615,1968$.

Gierasch, P. J., and R. M. Goody, The effect of dust on the temperature of the Martian atmosphere, J. Atmos. Sci., 29, 400, 1971.

Leovy, C. B., G. A. Briggs, and B. A. Smith, Mars atmosphere during the Mariner 9 extended mission: Television results, J. Geophys. Res., 78, this issue, 1973.

Masursky, H., et al., The new Mariner 9 map of Mars, Sky and Telescope, 44, 77, 1972.

Morrison, D., C. Sagan, and J. B. Pollack, Martian temperatures and thermal properties, Icarus, $11,36,1969$.

Neugebauer, G., G. Münch, H. H. Kieffer, S. C. Chase, Jr., and E. Miner, Mariner 1969 infrared radiometer results: Temperatures and thermal properties of the Martian surface, Astron. $J ., 76,719,1971$.

Pravda, Initial results of Mars probe analyzed, p. 3L, August 25, 1972.

Sharp, R. P., The surface of Mars, 2, Uncratered terrains, J. Geophys. Res., 76, 331, 1971.

Slipher, E. C., A Photographic Study of the Brighter Planets, p. 46, Northland Press, Flagstaff, Ariz., 1964.

Smith, S. A., and B. A. Smith, Diurnal and seasonal behavior of discrete white clouds on Mars, Icarus, 16, 509, 1972.

Wechsler, A. E., and P. E. Glaser, Pressure effects on postulated lunar materials, Icarus, 4, $355,1965$.

Zisk, S. H., M. H. Carr, H. Masursky, R. W. Shorthill, and T. W. Thompson, Lunar Apennine-Hadley regions: Geological implications of earth-based radar and infrared measurements, Science, 173, 808, 1971.

(Received January 4, 1973;

revised March 19, 1973.) 